\title{
A systematic review and meta-analysis of the use of resuscitative endovascular balloon occlusion of the aorta in the management of major exsanguination
}

\author{
B. L. S. Borger van der Burg ${ }^{1}$ - Thijs T. C. F. van Dongen ${ }^{1,2}$ (1) J. J. Morrison ${ }^{3}$. P. P. A. Hedeman Joosten ${ }^{1}$. J. J. DuBose ${ }^{4}$. \\ T. M. Hörer ${ }^{5}$ R. Hoencamp ${ }^{1,2,6}$
}

Received: 21 January 2018 / Accepted: 18 April 2018 / Published online: 21 May 2018

(c) The Author(s) 2018

\begin{abstract}
Background Circulatory collapse is a leading cause of mortality among traumatic major exsanguination and in ruptured aortic aneurysm patients. Approximately $40 \%$ of patients die before hemorrhage control is achieved. Resuscitative endovascular balloon occlusion of the aorta (REBOA) is an adjunct designed to sustain the circulation until definitive surgical or endovascular repair. A systematic review was conducted for the current clinical use of REBOA in patients with hemodynamic instability and to discuss its potential role in improving prehospital and in-hospital outcome.

Methods Systematic review and meta-analysis (1900-2017) using MEDLINE, Cochrane, EMBASE, Web of Science and Central and Emcare using the keywords "aortic balloon occlusion", "aortic balloon tamponade", "REBOA", and "Resuscitative Endovascular Balloon Occlusion" in combination with hemorrhage control, hemorrhage, resuscitation, shock, ruptured abdominal or thoracic aorta, endovascular repair, and open repair. Original published studies on human subjects were considered.

Results A total of 490 studies were identified; 89 met criteria for inclusion. Of the 1436 patients, overall reported mortality was $49.2 \%(613 / 1246)$ with significant differences $(p<0.001)$ between clinical indications. Hemodynamic shock was evident in $79.3 \%$, values between clinical indications showed significant difference $(p<0.001)$. REBOA was favored as treatment in trauma patients in terms of mortality. Pooled analysis demonstrated an increase in mean systolic pressure by almost $50 \mathrm{mmHg}$ following REBOA use.

Conclusion REBOA has been used in trauma patients and ruptured aortic aneurysm patients with improvement of hemodynamic parameters and outcomes for several decades. Formal, prospective study is warranted to clarify the role of this adjunct in all hemodynamic unstable patients.
\end{abstract}

Keywords Trauma Shock $\cdot$ Endovascular $\cdot$ Aortic balloon occlusion $\cdot$ REBOA

B. L. S. Borger van der Burg and Thijs T. C. F. van Dongen contributed equally to this manuscript; shared first author.

Electronic supplementary material The online version of this article (https://doi.org/10.1007/s00068-018-0959-y) contains supplementary material, which is available to authorized users.

Thijs T. C. F. van Dongen

ttcfvandongen@alrijne.nl

Extended author information available on the last page of the article

\section{Introduction}

Controlling catastrophic bleeding is the major life saving skill in trauma and vascular surgery. The revival of the tourniquet for management of extremity bleeding and massive transfusion protocols found their basis on the battlefields of Iraq and Afghanistan [1], however, difficult anatomical locations such as the neck, truncal and iliojunctional regions continue to represent challenges for prompt bleeding control. Truncal and junctional hemorrhage are often described within the trauma literature as non-survivable injuries contributing for almost $90 \%$ of catastrophic hemorrhage fatalities in the prehospital phase, in contrast to a $10 \%$ fatality rate in extremity injuries [2-4]. 
Bleeding from other non-compressible sites not related to trauma, such as the gastrointestinal tract, the post-partum uterus, a ruptured abdominal aortic aneurysm (rAAA), and traumatic disruption of thoracic, abdominal, or pelvic viscera can also represent similar challenges for prompt direct bleeding control and have traditionally been characterized as potentially non-survivable sources of hemorrhage. rAAA represents a classic source of non-traumatic major hemorrhage and has been associated with reported in-hospital mortality rates occurring in $30 \%$ of patients treated with EVAR and $42 \%$ of the patients undergoing open repair [5]. According to the Dutch Surgical Aneurysm Audit (DSAA) the number of patients that were treated in the Netherlands for a ruptured aortic aneurysm in 2013 was 293, with an in-hospital mortality of $35.3 \%$ [6]. It has also been demonstrated that approximately $25 \%$ of rAAAs undergo complete circulatory collapse before or during the procedure. The natural history of this uncontrolled hemorrhage has been shown to be cardiovascular collapse with consequent cerebral and myocardial hypoperfusion, ultimately leading to death $[7,8]$.

Endovascular balloon occlusion of the aorta is a technique where a compliant balloon is advanced into the aorta and then inflated, thereby obstructing flow into the distal circulation. This has the effect of increasing cardiac afterload and proximal aortic pressure, resulting in an increase in myocardial and cerebral perfusion [9]. This technique was first described by Hughes in 1954 when an intra-aortic balloon catheter tamponade was utilized in two moribund Korean War casualties with uncontrolled intra-abdominal hemorrhage [10].

In 1964, Heimbecker reported the first use of an aortic tampon for emergency control of a ruptured abdominal aneurysm [11].

Recent champions of the endovascular resuscitation and trauma management (EVTM) concept have re-introduced the concept of using resuscitative endovascular balloon occlusion of the aorta (REBOA) to modern clinical practice [12]. Two recent systematic analysis by these investigators have helped to consolidate a disparate evidence base [13, 14]. However, a clear mortality benefit has yet to be demonstrated and consequently, the REBOA concept is still not fully embedded as the standard of care in most hospitals.

Furthermore, the pioneering use of this hemorrhage control adjunct in the prehospital phase, despite early promising results $[15,16]$, has not been widely adopted. Recent terrorist threats in western countries have contributed to an increased awareness of the value for bleeding control in the earliest phases after injury, suggesting that the potential utilization of this REBOA in this setting warrants examination [17]. To date, however, a complete analysis of all international published literature has not been conducted. In particular, there is a need for an examination of focusing on differences between the main indications; trauma and nontrauma related major hemorrhage (including rAAA).

The primary aim of this systematic review was to examine the use of REBOA and the mortality and morbidity associated with REBOA in patients with hemodynamic instability due to major exsanguination from both traumatic and non-traumatic sources. The secondary aim was to provide an evidence-based rationale for optimal utilization of REBOA in the in-hospital phase and, ultimately contribute to the discussion of the potential for this technology to be more aggressively employed as an on-scene adjunct for control of major hemorrhage in both trauma and non-trauma settings.

\section{Methods}

The protocol for objectives, literature search strategies, inclusion and exclusion criteria, outcome measurements, and methods of statistical analysis was prepared a priori, according to the Preferred Reporting Items for Systematic Reviews and of Observational Studies in Epidemiology recommendations for study reporting and is described in this section $[18,19]$.

\section{Search strategy and selection criteria}

The MEDLINE, Cochrane, EMBASE, Web of Science and Central and Emcare databases were searched for relevant articles published from January 1900 to September 2017, using the Ovid medical search engine. The keywords used in the search were composed of combinations of "aortic balloon occlusion", "aortic balloon tamponade", "REBOA", "Resuscitative Endovascular Balloon Occlusion" in combination with hemorrhage control, hemorrhage, resuscitation, shock, ruptured abdominal, thoracic aorta, endovascular repair, open repair. The search was limited to original studies on human subjects (abstracts), published in English language journals.

\section{Inclusion and exclusion criteria, data extraction and outcomes of interest}

Three reviewers (B.B.B., T.D. and R.H.) independently screened the abstracts for suitability. Publications were excluded where non-ruptured aortic aneurysms were reported or ineligible study types (e.g., letters and reviews) were identified. Following abstract screening, the remaining publications were subjected to a full-text assessment. The full-text assessment consisted of two reviewers independently assessing the publication to determine suitability for inclusion. Level I-V evidence was considered, and particular attention was paid to the reporting of hemodynamic performance, balloon type, balloon deployment technique, 
complications, and mortality. During the full-text assessment, articles were excluded if little or no clinical data were reported on the subject of balloon occlusion. Where articles undergoing full-text review identified additional relevant studies that had not been previously identified, the additional relevant studies were also reviewed and included if eligible. If disagreement was encountered at any stage of the publication inclusion/exclusion process, the senior reviewer (RH) and a fourth independent reviewer (P.H.J) arbitrated a final decision.

\section{Quality assessment}

Studies were rated for the level of evidence provided according to criteria by the Centre for Evidence Based Medicine in Oxford. The risk of bias was assessed for each study using the Cochrane Collaboration's tool for assessing the risk of bias. This tool assesses six domains (selection, performance, detection, attrition, reporting, and other) and rates the risk of bias in each as "high", "low", or "unclear" [14]. The key characteristics of the eligible studies in terms of study type, clinical setting, aortic zone of occlusion, mortality, and morbidity were extracted as described earlier and summarized in a tabular format. These studies are the best evidence of mortality and morbidity associated with REBOA published up to 2017.

\section{Statistical analysis}

The categorical variables were analyzed by their absolute and relative frequencies in percentages. For the trauma cohort, we performed a meta-analysis to calculate the effect on systolic blood pressure and mortality. Only studies were REBOA was used as a primary means to control blood loss and contained an adequate control group, were used in the meta-analysis. The association between two categorical variables was calculated by applying the Pearson Chi-square test or $I^{2}$ test. We used with fixed effect and used $X^{2} / I^{2}$ for heterogeneity. The software package SPSS (24.0, IBM Corporation, Armonk, New York), was used for statistical analysis to achieve a combined outcome. In all cases, $p<0.05$ (twosided) was considered statistically significant.

\section{Results}

A total of 440 unique studies were identified and underwent abstract screening (Fig. 1), 107 of these studies were deemed appropriate for and underwent full-text review. Of these, 30 studies reported little or no clinical data, leaving 77 studies for inclusion following full-text review. Reference review of these studies identified an additional 53 eligible studies, which were not identified during the key word search.
These studies were predominantly older in publication date but were still eligible for inclusion. Therefore, after final quantitative synthesis, 89 articles were selected, including 28 case reports [11, 20-46], 25 case series [10, 47-71], and 36 (retrospective) cohort studies [12, 72-105]. Also 8 reviews were identified but not included in calculations, due to concern for double counting of unique cases [5, 13 , 14, 106-110]. Most studies were deemed to be at high risk of bias. In total, 1482 patients treated with REBOA were included in analysis (Table 1).

\section{Indications, hemodynamic instability and mortality}

REBOA has been used for the management of hemorrhage grouped in three major clinical indications: (1) traumatic abdominopelvic hemorrhage (18 studies), (2) hemorrhage arising from rAAA (50 studies) and, (3) miscellaneous causes such as post-partum, gastro-intestinal bleeding or exsanguination during pelvic surgery (21 studies). The REBOA concept was used in all studies as a hemorrhage control and resuscitation adjunct for prompt hemorrhage control.

Hemodynamic instability (transient or non-responding to fluid therapy) was present in $79.3 \%$ (1175/1482) of the total studied population. The studies with patients with established hemorrhagic shock [systolic blood pressure (SBP) below $90 \mathrm{mmHg}$ ] due to trauma $90.9 \%$ (791/870), rAAA 91.7\% (355/387) and other $12.9 \%$ (29/225) were respectively described. Overall reported mortality within the group that was hemodynamically stabilized with REBOA was $49.2 \%$ (613/1246). Reported mortality among studies concerning trauma was $63.0 \%(545 / 865)$. Meta-analysis revealed a significant difference in mortality $(p<0.001)$ of REBOA compared with the mortality of patients treated with other means. It showed a risk difference of 0.27 (0.14-0.49) favoring REBOA (Fig. 2). In studies regarding rAAA mortality was $39.1 \%(61 / 156)$ and for miscellaneous causes $3.1 \%$ (7/225). There were no episodes of mortality reported among studies on post-partum hemorrhage, gastrointestinal bleeding, or with the use of balloon occlusion during pelvic and sacral surgery. Additional information can be found in supplemental Tables 1, 2 and 3.

\section{The effects of REBOA}

The effect on SBP in hemodynamic instability, as seen in Table 2, was a significant increase of SBP by a mean of $78.9 \mathrm{mmHg}$ in trauma (Fig. 3), $56.1 \mathrm{mmHg}$ in rAAA and $52.4 \mathrm{mmHg}$ other types of patients in established hemorrhagic shock, among the 23 studies [10, 12, 22, 25, 29-31, $34,36,40,41,44,45,47,48,52,55,58,59,62,66,71$, $97,104,105,111]$ reporting pre-REBOA and post-REBOA SBP values. 


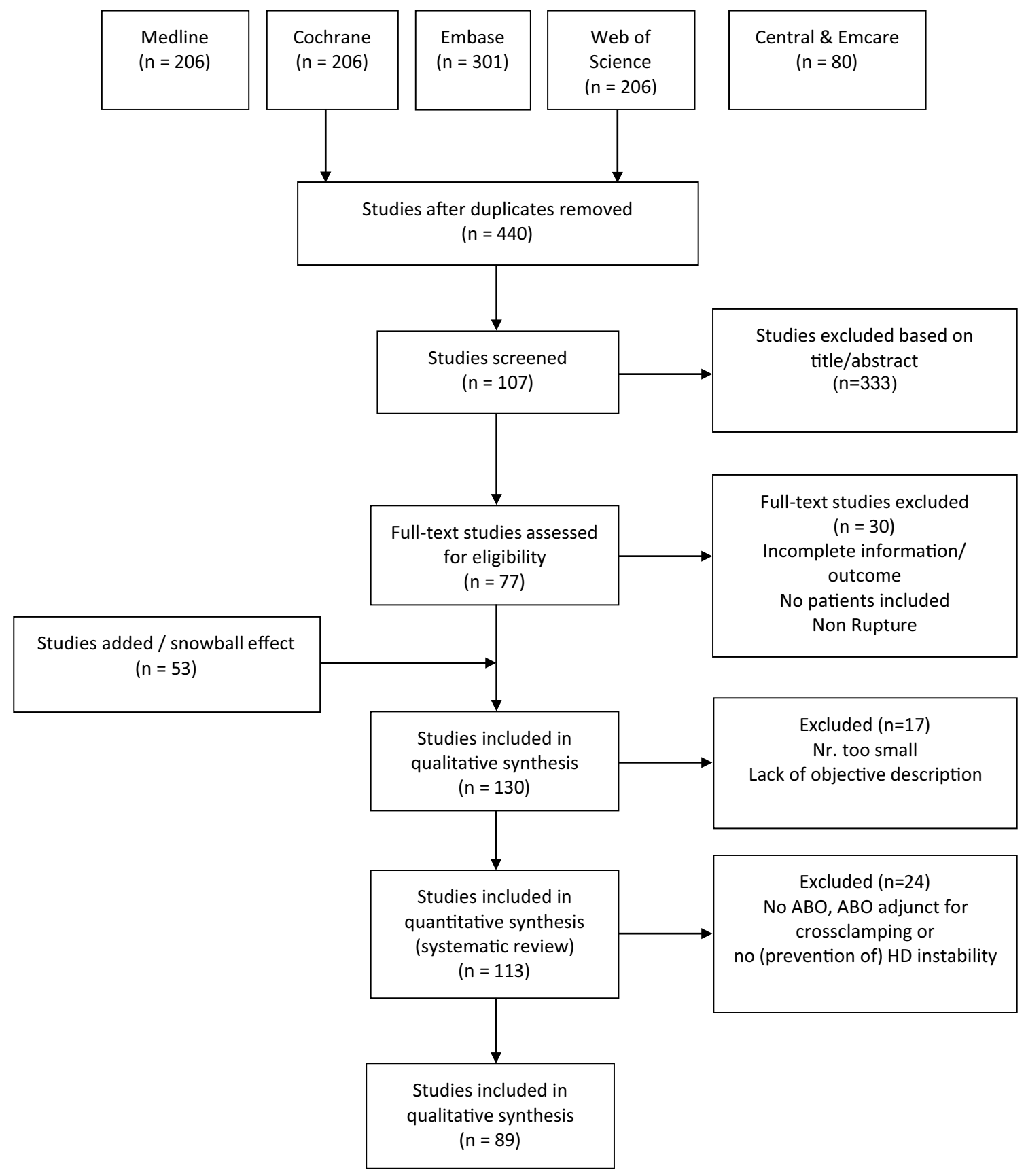

Fig. 1 PRISMA flow chart for the systematic review. $n$ indicates number, $A B O$ aortic balloon occlusion, $H D$ hemodynamic

REBOA balloon occlusion times were adequately reported in 35 studies [10, 12, 23, 25, 26, 29-34, 36, 38-41, $43-45,47,48,55,58,62,66,68-71,82,83,97,101,104$, 105]. Occlusion times within the zones of occlusion were described in 15 studies examining Zone I occlusion times, where 196 patients had a median occlusion time of $58.4 \mathrm{~min}$. Only three studies reported on occlusion in zone 2 , with a mean of $43.6 \mathrm{~min}$. The remaining nineteen studies examined 223 patients undergoing Zone III occlusion, with a median occlusion time of $55.2 \mathrm{~min}$.
Of the studies included for review, $81(92.0 \%)$ reported adequate data regarding arterial access and REBOA balloon deployment (Table 1). The femoral artery was the most commonly reported access site (96.8\% of the patients) for REBOA, followed by direct aortic access $(2.2 \%$ of the patients). Interestingly the largest study in our review, Norii et al $(n=452)$, did not report information on the access sites utilized [92]. Iatrogenic injuries related to REBOA use were $3.7 \%$ (32/870), $2.6 \%(10 / 387)$ and $5.3 \%$ (12/225) respectively on trauma, rAAA and miscellaneous causes $(p=0.21)$. 
Table 1 Included studies in systematic review

\begin{tabular}{|c|c|c|c|c|c|c|c|c|c|}
\hline Reference & Year & Study type & $\begin{array}{l}N \\
\text { Balloon (Tot) }\end{array}$ & $\begin{array}{l}\text { Aortic zone } \\
\text { placement }\end{array}$ & Shock & $\begin{array}{l}\text { Mortality (in- } \\
\text { hosp) }\end{array}$ & $\begin{array}{l}\text { AO related } \\
\text { iatrogenic } \\
\text { injury }\end{array}$ & Risk of bias & OXLE \\
\hline \multicolumn{10}{|l|}{ Case report (28) } \\
\hline Armour & 1978 & rAAA & 1 & I & $\mathrm{Y}$ & 0 & Nil & NA & V \\
\hline Bell-Thomas & 2003 & $\mathrm{PPH}$ & 1 & III & $\mathrm{Y}$ & 0 & Nil & NA & V \\
\hline Cakir & 2014 & rAAA & 1 & I & $\mathrm{Y}$ & 0 & Nil & NA & V \\
\hline D'Hondt & 2008 & $\begin{array}{l}\text { R aneurysma } \\
\text { aortobifem } \\
\text { graft }\end{array}$ & 1 & III & $\mathrm{Y}$ & 0 & Nil & NA & V \\
\hline Green & 2014 & $\begin{array}{l}\text { Trauma abd- } \\
\text { pel. hem }\end{array}$ & 1 & III & $\mathrm{N}$ & 0 & Nil & NA & V \\
\hline Harma & 2004 & PPH & 1 & III & $\mathrm{Y}$ & 0 & Nil & NA & V \\
\hline Heimbecker & 1964 & rAAA & 1 & III & $\mathrm{Y}$ & 0 & Nil & NA & V \\
\hline Hesse & 1962 & rAAA & 1 & III & $\mathrm{Y}$ & $1 / 1$ & Nil & NA & V \\
\hline Hill & 2010 & UGI bleeding & 1 & I & $\mathrm{Y}$ & 0 & Nil & NA & V \\
\hline Howard & 1972 & rAAA & 1 & III & $\mathrm{Y}$ & 0 & Nil & NA & V \\
\hline Karkos & 2001 & UGI bleeding & 1 & I & Y & 0 & Nil & NA & V \\
\hline Lai & 2008 & rAAA & 1 & I & $\mathrm{Y}$ & 0 & Nil & NA & V \\
\hline Lee & 2016 & GI bleeding & 1 & I & $\mathrm{Y}$ & 0 & Nil & NA & V \\
\hline Malina & 2005 & rAAA & 1 & I & $\mathrm{Y}$ & 0 & Nil & NA & V \\
\hline Masamoto & 2009 & $\mathrm{PPH}$ & 1 & III & $\mathrm{N}$ & 0 & Nil & NA & V \\
\hline Matsuoka & 2001 & $\begin{array}{c}\text { Trauma abd- } \\
\text { pel. Hem }\end{array}$ & 1 & I & $\mathrm{Y}$ & 0 & Nil & NA & V \\
\hline Menke & 2010 & $\begin{array}{l}\text { r-Para-anasto- } \\
\text { motic iliac } \\
\text { aneur }\end{array}$ & 1 & III & $\mathrm{Y}$ & 0 & Nil & NA & V \\
\hline Namura & 2001 & rAAA & 1 & III & $\mathrm{Y}$ & 0 & Nil & NA & V \\
\hline Ozgiray & 2009 & $\begin{array}{l}\text { Pelvic bleed- } \\
\text { ing peri-OK }\end{array}$ & 1 & III & $\mathrm{N}$ & 0 & Nil & NA & $\mathrm{V}$ \\
\hline Paull & 1995 & PPH & 1 & III & $\mathrm{N}$ & 0 & Nil & NA & V \\
\hline Pettersson & 2003 & $\begin{array}{l}\text { r. pseudo } \\
\text { aneurysma } \\
\text { aorta asc }\end{array}$ & 1 & I & $\mathrm{Y}$ & 0 & Nil & NA & V \\
\hline Schumacher & 2004 & rAAA & 1 & II & $\mathrm{Y}$ & 0 & Nil & NA & V \\
\hline Shigesato & 2015 & UGI & 1 & I & $\mathrm{Y}$ & 0 & Nil & NA & V \\
\hline Smith & 1972 & rAAA & 1 & III & $\mathrm{Y}$ & 0 & Nil & NA & V \\
\hline Soda & 2010 & $\begin{array}{l}\text { Pelvic bleed- } \\
\text { ing peri-OK }\end{array}$ & 1 & III & $\mathrm{N}$ & 0 & Nil & NA & $\mathrm{V}$ \\
\hline Uchida & 2014 & $\begin{array}{l}\text { Trauma abd- } \\
\text { pel. hem }\end{array}$ & 1 & III & $\mathrm{Y}$ & 0 & Nil & NA & V \\
\hline Wolf & 1986 & $\begin{array}{l}\text { Trauma abd- } \\
\text { pel. hem }\end{array}$ & 1 & III & $\mathrm{Y}$ & 0 & Nil & NA & $\mathrm{V}$ \\
\hline Xiong & 2014 & $\begin{array}{l}\text { Pelvic bleed- } \\
\text { ing peri-OK }\end{array}$ & 1 & III & $\mathrm{N}$ & 0 & Nil & NA & V \\
\hline Total & & & 28 & & $22 / 28$ & $1 / 28$ & 0 & & \\
\hline \multicolumn{10}{|l|}{ Case series (25) } \\
\hline Arthurs & 2006 & rAAA & 3 & I & $\mathrm{N}$ & $0 / 3$ & Nil & High & IV \\
\hline Brenner & 2013 & $\begin{array}{l}\text { Trauma abd- } \\
\text { pel. hem }\end{array}$ & 6 & Ix4, IIIx 2 & $\mathrm{Y}$ & $2 / 6$ & Nil & High & IV \\
\hline Delalieux & 2010 & rAAA & $1(18)$ & $\mathrm{U}$ & $\mathrm{Y}$ & $\mathrm{U}(7 / 18)$ & Nil & High & IV \\
\hline Greenberg & 2000 & rAAA & $2(3)$ & I & Y & $0 / 2$ & Nil & High & IV \\
\hline Guo & 2009 & rAAA & $4(26)$ & II/III & Y & $\mathrm{U}(7 / 26)$ & Nil & High & IV \\
\hline
\end{tabular}


Table 1 (continued)

\begin{tabular}{|c|c|c|c|c|c|c|c|c|c|}
\hline Reference & Year & Study type & $\begin{array}{l}N \\
\text { Balloon (Tot) }\end{array}$ & $\begin{array}{l}\text { Aortic zone } \\
\text { placement }\end{array}$ & Shock & $\begin{array}{l}\text { Mortality (in- } \\
\text { hosp) }\end{array}$ & $\begin{array}{l}\text { AO related } \\
\text { iatrogenic } \\
\text { injury }\end{array}$ & Risk of bias & OXLE \\
\hline Gupta & 1989 & $\begin{array}{c}\text { Trauma abd- } \\
\text { pel. Hem }\end{array}$ & 21 & I & $\mathrm{Y}$ & $14 / 21$ & $\begin{array}{l}1 \times \text { fem a } \\
\text { thrombosis }\end{array}$ & High & IV \\
\hline Hinchliffe & 2001 & rAAA & $2(20)$ & I & Y & $\mathrm{U}(9 / 20)$ & Nil & High & IV \\
\hline Hughes & 1954 & $\begin{array}{l}\text { Trauma abd- } \\
\text { pel. hem }\end{array}$ & 2 & I & $\mathrm{Y}$ & $2 / 2$ & Nil & High & IV \\
\hline Irahara & 2015 & $\begin{array}{l}\text { Trauma abd- } \\
\text { pel. hem }\end{array}$ & 14 & I & $\mathrm{Y}$ & $9 / 14$ & Nil & High & IV \\
\hline Lagana & 2006 & rAAA & $3(30)$ & II & $\mathrm{Y}$ & $\mathrm{U}(3 / 30)$ & NR & High & IV \\
\hline Lee & 2008 & rAAA & $3(52)$ & $\mathrm{U}$ & $\mathrm{Y}$ & $\mathrm{U}(28 / 52)$ & Nil & & IV \\
\hline Martinelli & 2010 & $\begin{array}{l}\text { Trauma abd- } \\
\text { pel. hem }\end{array}$ & 13 & III & $\mathrm{Y}$ & $7 / 13$ & $\begin{array}{l}\text { 1x fem a } \\
\text { thrombosis, } \\
\text { 1x balloon } \\
\text { rupture }\end{array}$ & High & IV \\
\hline Matsuda & 2003 & rAAA & $11(11)$ & III & $\mathrm{Y}$ & $3 / 11$ & $\begin{array}{l}\text { 3x bal. } \\
\text { rupture, } 2 \mathrm{x} \\
\text { embolic } \\
\text { complication }\end{array}$ & High & IV \\
\hline Mayer & 2009 & rAAA & 19 & I & $\mathrm{Y}$ & $\mathrm{U}$ & $\begin{array}{l}\text { 1x embolic } \\
\text { complication }\end{array}$ & High & IV \\
\hline $\mathrm{Ng}$ & 1977 & rAAA & 5 & III & $\mathrm{Y}$ & $3 / 5$ & Nil & High & IV \\
\hline Ogura & 2015 & $\begin{array}{l}\text { Trauma abd- } \\
\text { pel. hem }\end{array}$ & 35 & I & $\mathrm{Y}$ & $16 / 35$ & Nil & High & IV \\
\hline Ohki & 2000 & rAAA & 9 & I & $\mathrm{Y}$ & $\mathrm{U}$ & Nil & High & IV \\
\hline Philipsen & 2009 & rAAA & 12 & I & $\mathrm{Y}$ & $1 / 12$ & Nil & High & IV \\
\hline Sensenig & 1981 & rAAA & 3 & $\mathrm{I} / \mathrm{II} / \mathrm{I}$ & Y & $0 / 3$ & Nil & Low & IV \\
\hline Sovik & 2012 & $\mathrm{PPH}$ & 6 & III & $\mathrm{Y}$ & $0 / 6$ & $\begin{array}{l}\text { 1x Aortic } \\
\text { injury }\end{array}$ & High & IV \\
\hline Taheri & 1988 & rAAA & 2 & III & N/Y & $0 / 2$ & Nil & Low & IV \\
\hline Wang & 2013 & $\begin{array}{l}\text { Trauma abd- } \\
\text { pel. hem }\end{array}$ & 5 & III & $\mathrm{Y}$ & $\mathrm{U}$ & Nil & High & IV \\
\hline Xue-Song & 2010 & $\begin{array}{l}\text { Pelvic bleed- } \\
\text { ing peri-OK }\end{array}$ & 9 & III & $\mathrm{N}$ & $0 / 9$ & Nil & High & IV \\
\hline Yang & 2008 & $\begin{array}{l}\text { Pelvic bleed- } \\
\text { ing peri-OK }\end{array}$ & 12 & III & $\mathrm{N}$ & $0 / 12$ & Nil & High & IV \\
\hline Zhang & 2007 & $\begin{array}{l}\text { Pelvic bleed- } \\
\text { ing peri-OK }\end{array}$ & 5 & III & $\mathrm{N}$ & $0 / 5$ & Nil & High & IV \\
\hline Total & & & $207(341)$ & & $177 / 207$ & $57 / 161$ & 10 & & \\
\hline \multicolumn{10}{|c|}{ Cohort study (36) } \\
\hline Alsac & 2005 & rAAA & $1(37)$ & I & $\mathrm{Y}$ & $\mathrm{U}(14 / 37)$ & Nil & High & \\
\hline Anain & 2007 & rAAA & $12(40)$ & I & $\mathrm{Y}$ & $5 / 12$ & Nil & High & IV \\
\hline Carafiello & 2012 & rAAA & $4(42)$ & $\mathrm{U}$ & $\mathrm{Y}$ & $\mathrm{U}(13 / 42)$ & Nil & High & IV \\
\hline Coppi & 2006 & rAAA & $4(124)$ & II & $\mathrm{Y}$ & $\mathrm{U}(73 / 142)$ & Nil & High & IV \\
\hline Dalainas & 2006 & rAAA & 28 & II & $5 / 20$ & $8 / 28$ & Nil & High & IV \\
\hline Djavani G & 2011 & rAAA & $2(29)$ & $\mathrm{U}$ & $\mathrm{Y}$ & $1 / 2$ & Nil & High & IV \\
\hline DuBose & 2016 & Trauma & 46 (114) & $\begin{array}{l}\text { Ix33, IIx } 1, \\
\text { IIIx } 8\end{array}$ & $21 / 46$ & $13 / 46$ & $\begin{array}{l}\text { 2x embolism, } \\
\text { 1xpseudo } \\
\text { aneurysm }\end{array}$ & High & IV \\
\hline Gerassimidis & 2008 & rAAA & $2(41)$ & $\mathrm{U}$ & $\mathrm{Y}$ & $\mathrm{U}(15 / 41)$ & Nil & High & IV \\
\hline Holst & 2009 & rAAA & $23(90)$ & I & $\mathrm{Y}$ & $\begin{array}{l}\text { U }(24 / 90) \\
\quad(B O A \text { corr } \\
\text { sign,30d) }\end{array}$ & $\begin{array}{l}2 x \text { SMA } \\
\text { coverage }\end{array}$ & High & IV \\
\hline
\end{tabular}


Table 1 (continued)

\begin{tabular}{|c|c|c|c|c|c|c|c|c|c|}
\hline Reference & Year & Study type & $\begin{array}{l}N \\
\text { Balloon (Tot) }\end{array}$ & $\begin{array}{l}\text { Aortic zone } \\
\text { placement }\end{array}$ & Shock & $\begin{array}{l}\text { Mortality (in- } \\
\text { hosp) }\end{array}$ & $\begin{array}{l}\text { AO related } \\
\text { iatrogenic } \\
\text { injury }\end{array}$ & Risk of bias & OXLE \\
\hline Hörer & 2015 & Trauma/Other & 11 & $\begin{array}{l}\text { NA } \\
2 x \text { iatr, } 2 \text { gyn, } \\
7 \text { trauma }\end{array}$ & $\mathrm{Y}$ & $4 / 11$ & $\begin{array}{l}3 \times(2 \times \text { perf. } \\
\text { (iliaca/fem), } \\
\text { uncontroled } \\
\text { ECMO } \\
\text { removal }\end{array}$ & High & IV \\
\hline Hörer & 2017 & Trauma & 96 & $\begin{array}{l}\text { Ix86, IIx3, } \\
\text { IIIx3 }\end{array}$ & $43 / 65$ & $54 / 96$ & $\begin{array}{l}13 \text { (miscella- } \\
\text { neous) }\end{array}$ & High & IV \\
\hline Ioannidis & 2012 & rAAA & $1(20)$ & $\mathrm{U}$ & $\mathrm{Y}$ & U (10/20) & Nil & High & IV \\
\hline Karkos & 2008 & rAAA & $2(41)$ & $\mathrm{U}$ & $\mathrm{Y}$ & $\mathrm{U}(17 / 41)$ & Nil & High & IV \\
\hline Larzon & 2005 & rAAA & $13(41)$ & I & $\mathrm{Y}$ & $\mathrm{U}(14 / 41)$ & Nil & High & IV \\
\hline Low & 1986 & $\begin{array}{l}\text { Trauma } \\
\text { Abd-pelvic/ } \\
\text { rAAA//oth }\end{array}$ & $22(15 / 5 / 2)$ & I & $\mathrm{Y}$ & $\begin{array}{l}\text { 17/22 Overall } \\
13 / 15 \text { Trauma } \\
1 / 5 \text { rAAA } \\
2 / 2 \text { Other }\end{array}$ & $\begin{array}{l}\text { 5x perc access } \\
\text { failure, } 1 \mathrm{x} \\
\text { cut down } \\
\text { failure }\end{array}$ & High & IV \\
\hline Luo & 2013 & $\begin{array}{l}\text { Pelvic bleed- } \\
\text { ing peri-OK }\end{array}$ & 45 & III & $\mathrm{N}$ & $0 / 45$ & $\begin{array}{l}3 \mathrm{x} \text { fem a } \\
\text { thrombosis }\end{array}$ & High & IV \\
\hline Matsumara & 2017 & Trauma & 106 & $\begin{array}{l}\text { Ix99, IIx5, } \\
\text { IIIx2 }\end{array}$ & $\mathrm{Y}$ & $38 / 106$ & Nil & Low & III \\
\hline Mayer & 2012 & rAAA & $\begin{array}{l}62 \text { (268) } \\
\text { (EVAR only) }\end{array}$ & I & $\mathrm{Y}$ & U (48/268) & Nil & High & III \\
\hline Mehta 2005 & 2005 & rAAA & $7(30)$ & I & $\mathrm{Y}$ & $\mathrm{U}(7 / 30)$ & Nil & High & IV \\
\hline Mehta 2013 & 2013 & rAAA & 23 (136) & I & $\mathrm{Y}$ & $\mathrm{U}(32 / 136)$ & Nil & High & IV \\
\hline Moore & 2006 & rAAA & $\begin{array}{l}7 \text { (20) (EVAR } \\
\text { only) }\end{array}$ & II & Y & $1 / 7$ & Nil & High & IV \\
\hline Moore & 2015 & $\begin{array}{l}\text { Trauma abd- } \\
\text { pel. hem }\end{array}$ & 24 & Ix 19, IIIx5 & $\mathrm{Y}$ & $15 / 24$ & Nil & High & IV \\
\hline \multirow[t]{2}{*}{ Mukherjee } & 2014 & rAAA & $3(47)$ & I & $\mathrm{Y}$ & U (12/55) & & High & IV \\
\hline & 2014 & $\begin{array}{l}\text { rAAA (hybrid } \\
\text { group) }\end{array}$ & 8 & I & $3 / 8$ & $0 / 8$ & Nil & Low & IV \\
\hline Nedeau & 2012 & rAAA & $11(74)$ & $\mathrm{U}$ & $\mathrm{Y}$ & U (30/74) & Nil & High & IV \\
\hline Norii & 2015 & $\begin{array}{l}\text { Trauma abd- } \\
\text { pel. hem }\end{array}$ & 452 & $\mathrm{U}$ & $\mathrm{Y}$ & $343 / 452$ & Nil & High & III \\
\hline Ockert & 2007 & rAAA & $2(58)$ & $\mathrm{U}$ & Y & U (18/58) & Nil & High & IV \\
\hline $\begin{array}{l}\text { Peppelen- } \\
\text { bosch }\end{array}$ & 2005 & rAAA & $7(100)$ & II & $\mathrm{Y}$ & U (37/100) & Nil & High & IV \\
\hline Raux & 2015 & rAAA & $32(72)$ & II/III & Y & $22 / 32$ & Nil & Low & III \\
\hline Resch & 2003 & rAAA & $5(21)$ & U & Y & $\mathrm{U}(4 / 21)$ & Nil & High & IV \\
\hline Saito & 2014 & Blunt trauma & 24 & I-III & $\mathrm{Y}$ & $17 / 24$ & $\begin{array}{l}5 \times \text { failure of } \\
\text { infl } \\
1 \times \text { iliac injury }\end{array}$ & Low & IV \\
\hline Sarac & 2011 & rAAA & $3(32)$ & $\mathrm{U}$ & $\mathrm{Y}$ & $0 / 3$ & Nil & High & IV \\
\hline Starnes & 2010 & rAAA & $11(179)$ & $\mathrm{U}$ & $\mathrm{Y}$ & $7 / 11$ & Nil & High & IV \\
\hline Takagi & 2003 & $\begin{array}{l}\text { Aortic Arch } \\
\text { repair (rup- } \\
\text { tured only) }\end{array}$ & 8 & I & $\mathrm{Y}$ & $3 / 8$ & Nil & High & IV \\
\hline Tang & 2010 & $\begin{array}{l}\text { Pelvic bleed- } \\
\text { ing peri-OK }\end{array}$ & 120 & III & $\mathrm{N}$ & $0 / 120$ & $\begin{array}{l}\text { 3x fem a } \\
\text { embolism, } \\
5 x \text { puncture } \\
\text { site hema- } \\
\text { toma }\end{array}$ & High & III \\
\hline Veith & 2002 & rAAA & $10(31)$ & $\mathrm{U}$ & Y & $\mathrm{U}(3 / 31)$ & Nil & High & IV \\
\hline Veith & 2003 & rAAA & $10(36)$ & I & Y & $\mathrm{U}(26 / 36)$ & Nil & High & IV \\
\hline Total & & & 1247 (2659) & & $976 / 1216$ & $555 / 1057$ & 44 & & \\
\hline
\end{tabular}


Table 1 (continued)

\begin{tabular}{|c|c|c|c|c|c|c|c|c|c|}
\hline Reference & Year & Study type & $\begin{array}{l}N \\
\text { Balloon (Tot) }\end{array}$ & $\begin{array}{l}\text { Aortic zone } \\
\text { placement }\end{array}$ & Shock & $\begin{array}{l}\text { Mortality (in- } \\
\text { hosp) }\end{array}$ & $\begin{array}{l}\text { AO related } \\
\text { iatrogenic } \\
\text { injury }\end{array}$ & Risk of bias & OXLE \\
\hline $\begin{array}{l}\text { Grand total } \\
(n=89 \text { stud- } \\
\text { ies })\end{array}$ & & & $\begin{array}{c}1482(2960) \\
(50.1 \%)\end{array}$ & & $\begin{array}{c}1175 / 1482 \\
(79.3 \%)\end{array}$ & $\begin{array}{r}613 / 1246 \\
(49.2 \%)\end{array}$ & 54 & & \\
\hline
\end{tabular}

EVAR endovascular aneurysm repair, $N$ number, in-hosp in hospital, $A O$ "Arbeitsgemeinschaft für Osteosynthesefragen", $O X L E$ Oxford level of evidence, Art.Ins location of arterial incision, tech technique, Perc percutaneous, Occl occlusion, $S D$ standard deviation, $\Delta P \mathrm{mmHg}$ pressure difference in $\mathrm{mmHg}, r A A A$ ruptured abdominal aneurysm, abdpel hem abdominal/pelvic haemorrhage, $P P H$ post partum haemorrhage, (U)GI (upper) gastro intestinal, $O R$ operation room, $r$. ruptured, iatr/gyn iatrogenic/gynaecological, $Y$ yes, $N A$ not applicable, $U$ unknown, $n m$ not measurable, Ao aortal, $A x$ axillary, $B$ brachial, $C$ carotis, $F$ femora, (I)AOB (intra) aortic occlusion balloon, bal balloon, cath catheter, pts patients, fem $a$. femoral artery, def deflation, $p t(s)$ patient(s)

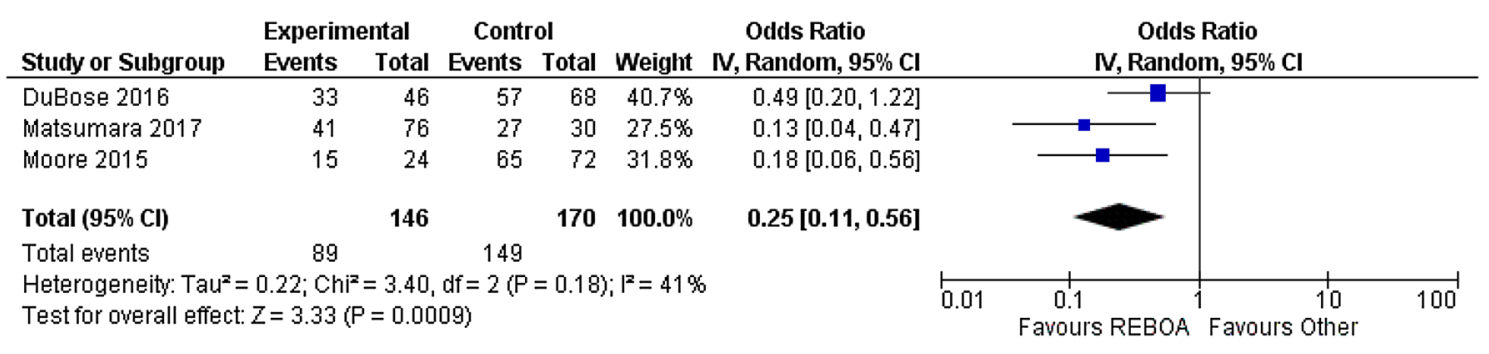

Fig. 2 Meta-analysis of mortality after use of REBOA in trauma. REBOA indicates resuscitative endovascular balloon occlusion of the aorta, $I V$ inverse variance, Random random effect, $C I$ confidence interval, $d f$ degrees of freedom, $P p$ value

\section{Discussion}

The current review is the largest systematic analysis of the literature on the subject of REBOA. A total of 89 studies, reporting on 1482 patients have been identified in the current published literature. The REBOA concept increases the SBP in hemorrhagic shock and is an adjunct for both endovascular and open repair in hemodynamic instability due to major exsanguination. The described iatrogenic injury rate related to REBOA is below $5 \%$.

\section{Central traumatic major exsanguination}

The most important question to answer is whether REBOA imparts a survival benefit in the in-hospital phase of care. Within the trauma literature, both non-survivors and survivors tended to have a higher burden of injury, presenting with gross physiologic derangements. The majority of studies lack a sound control group, with the exception of the studies by Norii et al. [92] and Moore et al. [88] In most studies, the study of Norii et al. [92] included, it was unclear whether REBOA was used in the context of a formal damage-control protocol, with consistent application, or whether it was used as a "last-ditch" attempt to salvage in hospital patients (e.g. ascending aortic ruptures) in civilian or military environment who were anticipated to have impending unfavorable outcome [14]. For this reason the study by Norii et al. [92], had to be excluded from the meta-analysis.

While our report suggests REBOA utilization has increased in recent years with results revealing a significant rise in blood pressure and a survival rate of approximately $40 \%$ with patients in shock (Table 2), the use of this adjunct is not universally embedded in hospital settings, not to mention the pioneering prehospital phase [15]. In the combat environment, major hemorrhage from central vascular injuries endures as the leading cause of death and most of the casualties decease before reaching a hospital [112-115]. REBOA can, therefore be an adjunct in the resuscitation of these major central/junctional hemorrhages in both the military as well as the civilian environment. Future research should focus on the usage of the REBOA concept in the prehospital phase, including selection and training of the right medical personnel for this important form of bleeding control.

\section{Ruptured acute aortic aneurysm}

The treatment of choice for rAAA depends on hemodynamic, morphological characteristics as well as the availability of an endovascular team trained for EVAR procedures. Historically, patients in profound hemodynamic 
Table 2 Mortality, occlusion time and rise of systolic blood pressure with application of REBOA

\begin{tabular}{|c|c|c|c|c|}
\hline & Number of studies & Total & Mortality & Unstable \\
\hline Trauma & 18 & 870 & $545 / 865(63.0 \%)$ & $791 / 870(90.9 \%)$ \\
\hline rAAA & 50 & 387 & $61 / 156(39.1 \%)$ & 355/387 (91.7\%) \\
\hline Other & 21 & 225 & $7 / 225(3.1 \%)$ & $29 / 225(12.9 \%)$ \\
\hline \multirow[t]{2}{*}{ Total } & 89 & 1482 & $613 / 1246(49.2 \%)$ & $1175 / 1482(79.3 \%)$ \\
\hline & Art. insertion & Acces tech & occlusion time & $\Delta P \mathrm{mmHg}$ \\
\hline \multirow[t]{5}{*}{ Trauma } & Aortal 0 & Percutaneous 310 & $11,953 / 230$ & Meta-analysis $(n=240)$ \\
\hline & Axillary 0 & & & \\
\hline & Brachial 1 & Cutdown 79 & $52.0 \mathrm{~min}$ & $79.8 \mathrm{mmHg}$ \\
\hline & Carotid 1 & & & \\
\hline & Femoral 388 & & & (pooled: $15,195 / 315$ ) \\
\hline \multirow[t]{5}{*}{ rAAA } & Aortal 7 & Percutaneous 221 & $436 / 13$ & $898 / 16$ \\
\hline & Axillary 3 & & & \\
\hline & Brachial 2 & Cutdown 99 & $33.5 \mathrm{~min}$ & $56.1 \mathrm{mmHg}$ \\
\hline & Carotid 0 & & & \\
\hline & Femoral 312 & & & \\
\hline \multirow[t]{5}{*}{ Other } & Aortal 8 & Percutaneous 165 & $10,319 / 159$ & $681 / 13$ \\
\hline & Axillary 0 & & & \\
\hline & Brachial 0 & Cutdown 56 & $64.9 \mathrm{~min}$ & $52.4 \mathrm{mmHg}$ \\
\hline & Carotid 0 & & & \\
\hline & Femoral 217 & & & \\
\hline \multirow[t]{6}{*}{ Total (pooled) } & Aortal 15 & Percutaneous 696 & $22,708 / 432$ & $16,773 / 344$ \\
\hline & Axillary 3 & & & \\
\hline & Brachial 3 & Cutdown 234 & $56.5 \mathrm{~min}$ & $48.8 \mathrm{mmHg}$ \\
\hline & Carotid 1 & & & \\
\hline & Femoral 669 & & & \\
\hline & Art. insertion & Mean occlusion time & & Number of studies \\
\hline \multirow[t]{3}{*}{ Trauma } & Zone I & $11,111 / 190=58.5 \mathrm{~min}$ & & 8 \\
\hline & Zone II & $270 / 6=45.0 \mathrm{~min}$ & & 2 \\
\hline & Zone III & $2175 / 32=68.0 \mathrm{~min}$ & & 8 \\
\hline \multirow[t]{3}{*}{ rAAA } & Zone I & $90 / 3=30.0 \mathrm{~min}$ & & 2 \\
\hline & Zone II & $30 / 1=30.0 \mathrm{~min}$ & & 2 \\
\hline & Zone III & $31 / 2=15.5 \mathrm{~min}$ & & 2 \\
\hline \multirow[t]{3}{*}{ Other } & Zone I & $205 / 4=51.3 \mathrm{~min}$ & & 4 \\
\hline & Zone II & 0 & & 0 \\
\hline & Zone III & $10,114 / 189=53.5 \mathrm{~min}$ & & 10 \\
\hline \multirow[t]{3}{*}{ Total } & Zone I & $11,496 / 197=58.4 \mathrm{~min}$ & & 15 \\
\hline & Zone II & $305 / 7=43.6 \mathrm{~min}$ & & 3 \\
\hline & Zone III & $12,320 / 223=55.2 \mathrm{~min}$ & & 20 \\
\hline
\end{tabular}

REBOA indicates resuscitative endovascular balloon occlusion of the aorta, $r A A A$ ruptured abdominal aneurysm, Art.Ins location of arterial incision, tech technique, $\min$ minutes, $\Delta P$ rise of systolic blood pressure in $\mathrm{mmHg}$

shock have been deemed not suitable for endovascular approach, because of the extra time involved in measuring and preparing for an endovascular procedure. The use of REBOA in these patients may extend the period needed to assemble the necessary staff for endovascular repair. It may even have potential application in the pre- or inter-hospital setting, to bridge the transport time to an endovascular center with sufficient expertise.

Three RCT's have studied the optimal treatment for ruptured aneurysms, EVAR or open [116-120]. In the reported results of the ECAR and AJAX trials, EVAR was found to be equal to open surgical repair in terms of 30 day and 1-year 


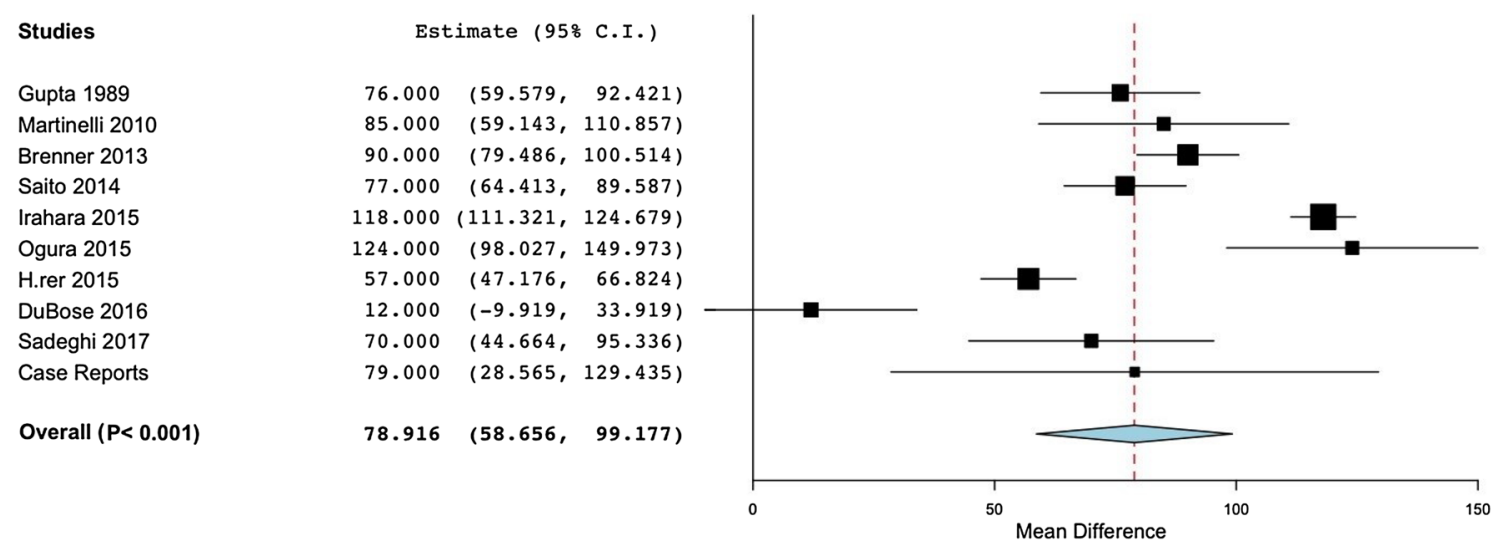

Fig. 3 Meta-analysis of rise in SBP after REBOA use in trauma. $S B P$ indicates systolic blood pressure in mmHg, REBOA resuscitative endovascular balloon occlusion of the aorta, $C I$ confidence interval, $P p$ value

mortality. In contrast, the IMPROVE trial concluded that a strategy of endovascular repair was not associated with significant reduction in either 30-day mortality or cost. The 2014 Cochrane review on this subject concluded that, based on available data at the time of analysis, there is no difference in the 30-day mortality outcomes between EVAR and open repair [121]. It is important to note, however, that none of the presently available trials have randomized high-risk patients with ruptured aneurysms, particularly those in shock or with low blood pressure [116]. These patients potentially represent the population most likely to benefit from early aortic balloon occlusion, possibly even in the pre-hospital phase or in the emergency department. This patient subset warrants further examination.

\section{Miscellaneous causes of major hemorrhage}

Miscellaneous causes of significant hemorrhage, such as post-partum hemorrhage, gastro-intestinal bleeding or exsanguination during pelvic surgery, are also likely to benefit from early direct bleeding control to prevent the onset of the lethal triad (hypothermia, acidosis and coagulopathy). While the data on this subset of patients is limited, our observed mortality rate of lower than 5\% among REBOA patients is suggestive that this technology has potentially important applications for these indications. Since shock was not presented in the studies regarding PPH, GI bleeding or pelvic surgery, additional examination of these patient subgroups is warranted.

\section{Limitations}

While our review represents the largest of such effort in the medical literature, it does possess several limitations that must be addressed. The major limitation of the current review is the quality of the available evidence, which is limited. The risk of population bias in this systematic review is inevitable, to minimize best possible effects of heterogeneity and cohort overlap we provided a narrative description of prevalence and characteristics. A recent published review of Gamberini et al. [122] contained several papers that are not mentioned in our study [123-133], most of them based on the Japanese trauma registry. It was not possible to determine how much overlap there was with the studies we included from the Japanese registry. To prevent bias by double patient inclusion, these studies were not included in this analysis. The majority of the evidence identified consists of case reports and case series (Grade IV, V evidence) with only limited cohort studies and reviews identified (Grade II, III evidence). Most studies are at significant risk of bias, underestimating the true mortality and morbidity (including iatrogenic injuries). These findings should prompt efforts to improve on this by stimulating formal prospective evaluation.

REBOA is a potential lifesaving adjunct for resuscitation but prolonged occlusion of the aorta can lead to organ failure due to resulting ischemia-reperfusion injury. Consensus regarding safe occlusion times is not yet reached although animal studies indicate an optimal duration of $30 \mathrm{~min}$. Occlusion time of $60 \mathrm{~min}$ or above is associated with an increased mortality [134, 135]. For prolonged field care or surgery, intermittent or partial occlusion could be a solution [71]. Three studies reported on occlusion times in zone 2 [40, 104, 111]. When the exact location of the bleeding is known, control in this zone could be needed in specific circumstances. The use of zone 2 as deployment zone is usually not recommended [136].

Prospective data collection is underway in the form of an American Association for the Surgery of Trauma sponsored observation study (AORTA), the European registry (ABOT), the Japanese registry (DIRECT-IABO) and the UK-REBOA Randomized Control Trial which should 
permit the consistent capture and reporting of REBOAspecific data such as indications, hemodynamic performance, outcome, cause of death, and morbidity [137-140].

Growing experience with the REBOA concept has proven its value in (acute) aortic repair, and expanding experience is gathering in the clinical implementation of this technology in major hemorrhage for trauma indications. Ongoing research should provide a much-needed higher quality of data in the coming years. These maturing experiences should yield improved data on the degree of hemorrhagic shock, specifics of the setting of use (pre- and in-hospital), the influence of use on organ failure, associated blood product requirements, encountered iatrogenic injuries and the specific techniques utilized for deployment (balloon occlusion time; full/partial /intermittent inflation approaches) [12, 104, 141].

\section{Conclusion}

The REBOA concept has been used as an effective early hemorrhage control and resuscitation adjunct following traumatic abdominopelvic hemorrhage, hemorrhage arising from rAAA and significant bleeding from miscellaneous causes (post-partum, gastro-intestinal bleeding or exsanguination during pelvic surgery). Once placed in the correct aortic zone it has been shown to effectively raise the SBP in patients in major hemorrhagic shock. Despite these documented hemodynamic improvements, however, there remains a need for improved evidence supporting mortality benefit following REBOA use in the setting of traumatic hemorrhage. Presently available data, however, continues to suggest a potential value for REBOA use in austere environments or for patients in extremis that cannot be ignored. For this specific subset of patients, where the traditional alternative is likely death, the adage "do you need a RCT to prove that a parachute works" may prove worthy of further discussion. A feasibility study to setup a formal training program for obtaining vascular access and REBOA placement by non-vascular medical specialists has recently been conducted by this study group. This systematic review underscores that solid prospective evaluation of the REBOA concept remains a significant requirement for the establishment of optimal guidelines for REBOA employment in the management of hemorrhagic shock in any phase of medical care.

Acknowledgements We thank J.W. Schoones (Walaeus Library, Leiden University Medical Center) for his methodological support.

Funding This research did not receive any specific grant from funding agencies in the public, commercial, or not-for-profit sectors.
Conflict of interest All authors declared that they have no conflict of interest.

Open Access This article is distributed under the terms of the Creative Commons Attribution 4.0 International License (http://creativeco mmons.org/licenses/by/4.0/), which permits unrestricted use, distribution, and reproduction in any medium, provided you give appropriate credit to the original author(s) and the source, provide a link to the Creative Commons license, and indicate if changes were made.

\section{References}

1. Blackbourne LH, Baer DG, Eastridge BJ, Kheirabadi B, Bagley S, Kragh JF Jr, et al. Military medical revolution: prehospital combat casualty care. J Trauma Acute Care Surg. 2012;73(6 Suppl 5):S372-7. https://doi.org/10.1097/TA.0b013e318275566 2.

2. Eastridge BJ, Mabry RL, Seguin P, Cantrell J, Tops T, Uribe P, et al. Death on the battlefield (2001-2011): implications for the future of combat casualty care. J Trauma Acute Care Surg. 2012;73(6 Suppl 5):S431-7. https://doi.org/10.1097/TA.0b013 e3182755dcc.

3. Hoencamp R, Vermetten E, Tan EC, Putter H, Leenen LP, Hamming JF. Systematic review of the prevalence and characteristics of battle casualties from NATO coalition forces in Iraq and Afghanistan. Injury. 2014;45(7):1028-34. https://doi. org/10.1016/j.injury.2014.02.012.

4. van Dongen TT, Idenburg FJ, Tan EC, Rasmussen TE, Hamming JF, Leenen LP, et al. Combat related vascular injuries: Dutch experiences from a role $2 \mathrm{MTF}$ in Afghanistan. Injury. 2016;47(1):94-8. https://doi.org/10.1016/j.injury.2015.08.030.

5. Antoniou GA, Georgiadis GS, Antoniou SA, Pavlidis P, Maras D, Sfyroeras GS, et al. Endovascular repair for ruptured abdominal aortic aneurysm confers an early survival benefit over open repair. J Vasc Surg. 2013;58(4):1091-105. https://doi. org/10.1016/j.jvs.2013.07.109.

6. Lijftogt N, Elsman BH, Wilschut ED, Geelkerken R, Legemate DA, Vahl AC, et al. The Dutch Surgical Aneurysm Audit (DSAA): 1 year of registration in the Netherlands. 2013.

7. Berland TL, Veith FJ, Cayne NS, Mehta M, Mayer D, Lachat M. Technique of supraceliac balloon control of the aorta during endovascular repair of ruptured abdominal aortic aneurysms. J Vasc Surg. 2013;57(1):272-5. https://doi.org/10.1016/j. jvs.2012.09.001.

8. Peitzman AB, Billiar TR, Harbrecht BG, Kelly E, Udekwu AO, Simmons RL. Hemorrhagic shock. Curr Probl Surg. 1995;32(11):925-1002.

9. Stannard A, Eliason JL, Rasmussen TE. Resuscitative endovascular balloon occlusion of the aorta (REBOA) as an adjunct for hemorrhagic shock. J Trauma. 2011;71(6):1869-72. https://doi. org/10.1097/TA.0b013e31823fe90c.

10. Hughes CW. Use of an intra-aortic balloon catheter tamponade for controlling intra-abdominal hemorrhage in man. Surgery. 1954;36(1):65-8.

11. Heimbecker RO. An aortic tampon for emergency control of ruptured abdominal aneurysm. Can Med Assoc J. 1964;91:1024-5.

12. Horer TM, Skoog P, Pirouzram A, Nilsson KF, Larzon T. A small case series of aortic balloon occlusion in trauma: lessons learned from its use in ruptured abdominal aortic aneurysms and a brief review. Eur J Trauma Emerg Surg. 2016;42(5):585-92. https:// doi.org/10.1007/s00068-015-0574-0. 
13. Karkos CD, Papadimitriou CT, Chatzivasileiadis TN, Kapsali NS, Kalogirou TE, Giagtzidis IT, et al. The impact of aortic occlusion balloon on mortality after endovascular repair of ruptured abdominal aortic aneurysms: a meta-analysis and meta-regression analysis. Cardiovasc Intervent Radiol. 2015;38(6):1425-37. https://doi.org/10.1007/s00270-015-1132-1.

14. Morrison JJ, Galgon RE, Jansen JO, Cannon JW, Rasmussen TE, Eliason JL. A systematic review of the use of resuscitative endovascular balloon occlusion of the aorta in the management of hemorrhagic shock. J Trauma Acute Care Surg. 2016;80(2):324-34.

15. Manley JD, Mitchell BJ, DuBose JJ, Rasmussen TE. A modern case series of resuscitative endovascular balloon occlusion of the aorta (REBOA) in an out-of-hospital, combat casualty care setting. J Spec Oper Med.17(1):1-8.

16. Reva VA, Horer TM, Makhnovskiy AI, Sokhranov MV, Samokhvalov IM, DuBose JJ. Field and en route resuscitative endovascular occlusion of the aorta: a feasible military reality? J Trauma Acute Care Surg. 2017;83(1 Suppl 1):S170-S6. https://doi. org/10.1097/TA.0000000000001476.

17. American College of Surgeons. Beeldingcontrolorg. 2017. Accessed July 82017.

18. Moher D, Liberati A, Tetzlaff J, Altman DG, Group P. Preferred reporting items for systematic reviews and meta-analyses: the PRISMA statement. J Clin Epidemiol. 2009;62(10):1006-12. https://doi.org/10.1016/j.jclinepi.2009.06.005.

19. Stroup DF, Berlin JA, Morton SC, Olkin I, Williamson GD, Rennie D, et al. Meta-analysis of observational studies in epidemiology: a proposal for reporting. Meta-analysis of observational studies in epidemiology (MOOSE) group. JAMA. 2000;283(15):2008-12.

20. Armour RH, Clifton MA, Marsh CH. Balloon catheter contol of a ruptured abdominal aortic aneurysm in a patient with Cullen's sign. Br J Surg. 1978;65(5):350.

21. Bell-Thomas SM, Penketh RJ, Lord RH, Davies NJ, Collis R. Emergency use of a transfemoral aortic occlusion catheter to control massive haemorrhage at caesarean hysterectomy. BJOG. 2003;110(12):1120-2.

22. Cakir H, Acar G, Pala S. Fast track occlusion of aorta with atrial septal defect sizing balloon. Catheter Cardiovasc Interv. 2014;83(7):1182-4. https://doi.org/10.1002/ccd.25379.

23. D'Hondt M, Van Lysebeth L, De Smul G, Wallaert P, Ceuppens $\mathrm{H}$. Emergency balloon sealing of a ruptured giant anastomotic aneurysm of the groin. Acta Chir Belg. 2008;108(6):741-3.

24. Green KD, Waked A, Majeed U, Fudim M, Robbins MA, Crenshaw M, et al. Percutaneous management of RetroFlex 3 balloon rupture and separation of the Edwards Sapien delivery system. Tex Heart Inst J. 2014;41(6):641-4. https://doi.org/10.14503/ THIJ-13-3447.

25. Harma M, Harma M, Kunt AS, Andac MH, Demir N. Balloon occlusion of the descending aorta in the treatment of severe post-partum haemorrhage. Aust $\mathrm{N}$ Z J Obstet Gynaecol. 2004;44(2):170-1. https://doi.org/10.1111/j.1479828X.2004.00181.x.

26. Hesse FG, Kletschka HD. Rupture of abdominal aortic aneurysm: control of hemorrhage by intraluminal balloon tamponade. Ann Surg. 1962;155:320-2.

27. Hill SJ, Zarroug AE, Ricketts RR, Veeraswamy R. Bedside placement of an aortic occlusion balloon to control a ruptured aorto-esophageal fistula in a small child. Ann Vasc Surg. 2010;24(6):822 e7-9. https://doi.org/10.1016/j.avsg.2009.12.016.

28. Howard ER, Young AE. Control of aortic haemorrhage by balloon catheter. Br Med J. 1971;3(5767):161.
29. Karkos CD, Bruce IA, Lambert ME. Use of the intra-aortic balloon pump to stop gastrointestinal bleeding. Ann Emerg Med. 2001;38(3):328-31. https://doi.org/10.1067/mem.2001.114408.

30. Lai CH, Wu HY, Kan CD. Resuscitation for an octogenarian with ruptured abdominal aortic aneurysm using endovascular balloon. Am J Emerg Med. 2008;26(8):967-3. https://doi.org/10.1016/j. ajem.2008.02.008

31. Lee J, Kim K, Jo YH, Lee JH, Kim J, Chung H, et al. Use of resuscitative endovascular balloon occlusion of the aorta in a patient with gastrointestinal bleeding. Clin Exp Emerg Med. 2016;3(1):55-8. https://doi.org/10.15441/ceem.15.006.

32. Malina M, Veith F, Ivancev K, Sonesson B. Balloon occlusion of the aorta during endovascular repair of ruptured abdominal aortic aneurysm. J Endovasc Ther. 2005;12(5):556-9. https://doi. org/10.1583/05-1587.1.

33. Masamoto H, Uehara H, Gibo M, Okubo E, Sakumoto K, Aoki Y. Elective use of aortic balloon occlusion in cesarean hysterectomy for placenta previa percreta. Gynecol Obstet Invest. 2009;67(2):92-5. https://doi.org/10.1159/000164685.

34. Matsuoka S, Uchiyama K, Shima H, Ohishi S, Nojiri Y, Ogata $\mathrm{H}$. Temporary percutaneous aortic balloon occlusion to enhance fluid resuscitation prior to definitive embolization of posttraumatic liver hemorrhage. Cardiovasc Intervent Radiol. 2001;24(4):274-6.

35. Menke V, Castenmiller PH, Versteijlen RJ, Van der Laan L. Stent grafting a ruptured para-anastomotic iliac aneurysm. Vasc Endovascular Surg. 2010;44(6):479-82. https://doi.org/10.1177/15385 74410368142 .

36. Namura O, Kanazawa H, Yoshiya K, Nakazawa S, Yamazaki Y. Successful surgical treatment of a ruptured abdominal aortic aneurysm without homologous blood transfusion in a Jehovah's Witness: report of a case. Surg Today. 2001;31(10):912-4. https ://doi.org/10.1007/s005950170034.

37. Ozgiray E, Cagli S, Zileli M, Cinar C, Oran I. Occlusion of the abdominal aorta by balloon dilation catheter assisting surgical excision of a sacrum chordoma: case report. Turk Neurosurg. 2009;19(3):265-8.

38. Paull JD, Smith J, Williams L, Davison G, Devine T, Holt M. Balloon occlusion of the abdominal aorta during caesarean hysterectomy for placenta percreta. Anaesth Intensive Care. 1995;23(6):731-4.

39. Pettersson G, Nores M, Gillinov AM. Transfemoral control of ruptured aortic pseudoaneurysm at aortic root reoperation. Ann Thorac Surg. 2004;77(1):311-2.

40. Schumacher H, Schwarzbach M, Bockler D, Megges I, Allenberg J-R. Successful endovascular emergency repair of a ruptured abdominal aortic aneurysm under cardiovascular resuscitation. [German: Erfolgreiche endovaskulare notfallversorgung eines rupturierten infrarenalen aortenaneurysmas unter reanimationsbedingungen]. Gefasschirurgie. 2004;9(4):324-30.

41. Shigesato S, Shimizu T, Kittaka T, Akimoto H. Intra-aortic balloon occlusion catheter for treating hemorrhagic shock after massive duodenal ulcer bleeding. Am J Emerg Med. 2015;33(3):473 e1-2. https://doi.org/10.1016/j.ajem.2014.01.024.

42. Smith FG. Emergency control of ruptured abdominal aortic aneurysm by transaxillary balloon catheter. Vasc Surg. 1972;6(2):79-84.

43. Soda H, Kainuma O, Yamamoto H, Nagata M, Takiguchi N, Ikeda A, et al. Giant intrapelvic solitary fibrous tumor arising from mesorectum. Clin J Gastroenterol. 2010;3(3):136-9. https ://doi.org/10.1007/s12328-010-0146-0.

44. Uchida K, Homma H, Oda J, Yukioka T, Nagai N, Mishima S, et al. Hemostasis with emergently modified application of intraaortic balloon occlusion in a patient with impending cardiac 
arrest following blunt proximal thigh amputation. Acute Med Surg. 2015;2(1):69-71. https://doi.org/10.1002/ams2.62.

45. Wolf RK, Berry RE. Transaxillary intra-aortic balloon tamponade in trauma. J Vasc Surg. 1986;4(1):95-7.

46. Xiong Y, Lang Y, Tu C, Duan H. Pelvis metastasis from primary choroidal melanoma: a case report. Onco Targets Ther. 2014;7:2107-10. https://doi.org/10.2147/OTT.S71062.

47. Arthurs Z, Starnes B, See C, Andersen C. Clamp before you cut: proximal control of ruptured abdominal aortic aneurysms using endovascular balloon occlusion-case reports. Vasc Endovascular Surg. 2006;40(2):149-55. https://doi.org/10.1177/15385 7440604000210

48. Brenner ML, Moore LJ, DuBose JJ, Tyson GH, McNutt MK, Albarado RP, et al. A clinical series of resuscitative endovascular balloon occlusion of the aorta for hemorrhage control and resuscitation. J Trauma Acute Care Surg. 2013;75(3):506-11. https:// doi.org/10.1097/TA.0b013e31829e5416.

49. Delalieux S, Hendriks JM, Lauwers P, Schwagten V, Jorens P, d'Archambeau O, et al. Emergency endovascular aneurysm repair for ruptured abdominal aortic aneurysms: an institutional experience. Acta Chir Belg. 2010;110(3):272-4.

50. Greenberg RK, Srivastava SD, Ouriel K, Waldman D, Ivancev K, Illig KA, et al. An endoluminal method of hemorrhage control and repair of ruptured abdominal aortic aneurysms. J Endovasc Ther. 2000;7(1):1-7. https://doi.org/10.1177/152660280000700 101.

51. Guo W, Zhang HP, Liu XP, Yin T, Jia X, Liang FQ, et al. Endovascular repair: alternative treatment of ruptured abdominal aortic aneurysm. Chin Med J (Engl). 2009;122(15):1728-31.

52. Gupta BK, Khaneja SC, Flores L, Eastlick L, Longmore W, Shaf$\tan \mathrm{GW}$. The role of intra-aortic balloon occlusion in penetrating abdominal trauma. J Trauma. 1989;29(6):861-5.

53. Hinchliffe RJ, Yusuf SW, Macierewicz JA, MacSweeney ST, Wenham PW, Hopkinson BR. Endovascular repair of ruptured abdominal aortic aneurysm-a challenge to open repair? Results of a single centre experience in 20 patients. Eur J Vasc Endovasc Surg. 2001;22(6):528-34. https://doi.org/10.1053/ ejvs.2001.1513.

54. Ingoldby CJ, Case WG, Primrose JN. Aortocaval fistulas and the use of transvenous balloon tamponade. Ann R Coll Surg Engl. 1990;72(5):335-8.

55. Irahara T, Sato N, Moroe Y, Fukuda R, Iwai Y, Unemoto K. Retrospective study of the effectiveness of Intra-Aortic Balloon Occlusion (IABO) for traumatic haemorrhagic shock. World J Emerg Surg. 2015;10(1):1. https://doi.org/10.1186/1749-7922-10-1.

56. Lagana D, Carrafiello G, Mangini M, Fontana F, Caronno R, Castelli P, et al. Emergency endovascular treatment of abdominal aortic aneurysms: feasibility and results. Cardiovasc Intervent Radiol. 2006;29(2):241-8. https://doi.org/10.1007/s0027 0-005-0080-6.

57. Lee RW, Rhodes JM, Singh MJ, Davies MG, Wolford HY, Diachun $\mathrm{C}$, et al. Is there a selection bias in applying endovascular aneurysm repair for rupture? Ann Vasc Surg. 2008;22(2):215-20. https://doi.org/10.1016/j.avsg.2007.12.006.

58. Martinelli T, Thony F, Declety P, Sengel C, Broux C, Tonetti J, et al. Intra-aortic balloon occlusion to salvage patients with lifethreatening hemorrhagic shocks from pelvic fractures. J Trauma. 2010;68(4):942-8. https://doi.org/10.1097/TA.0b013e3181c4057 9.

59. Matsuda H, Tanaka Y, Hino Y, Matsukawa R, Ozaki N, Okada $\mathrm{K}$, et al. Transbrachial arterial insertion of aortic occlusion balloon catheter in patients with shock from ruptured abdominal aortic aneurysm. J Vasc Surg. 2003;38(6):1293-6. https://doi. org/10.1016/S0741.

60. Mayer D, Pfammatter T, Rancic Z, Hechelhammer L, Wilhelm $\mathrm{M}$, Veith FJ, et al. 10 years of emergency endovascular aneurysm repair for ruptured abdominal aortoiliac aneurysms: lessons learned. Ann Surg. 2009;249(3):510-5. https://doi.org/10.1097/ SLA.0b013e31819a8b65.

61. Ng AC, Ochsner EC. Use of Fogarty catheter tamponade for ruptured abdominal aortic aneurysms. AJR Am J Roentgenol. 1977;128(1):31-3. https://doi.org/10.2214/ajr.128.1.31.

62. Ogura T, Lefor AT, Nakano M, Izawa Y, Morita H. Nonoperative management of hemodynamically unstable abdominal trauma patients with angioembolization and resuscitative endovascular balloon occlusion of the aorta. J Trauma Acute Care Surg. 2015;78(1):132-5. https://doi.org/10.1097/TA.0000000000 000473.

63. Ohki T, Veith FJ. Endovascular grafts and other image-guided catheter-based adjuncts to improve the treatment of ruptured aortoiliac aneurysms. Ann Surg. 2000;232(4):466-79.

64. Philipsen TE, Hendriks JM, Lauwers P, Voormolen M, d'Archambeau O, Schwagten V, et al. The use of rapid endovascular balloon occlusion in unstable patients with ruptured abdominal aortic aneurysm. Innovations (Phila). 2009;4(2):74-9. https://doi.org/10.1097/IMI.0b013e3181a00bc9.

65. Sensenig DM. Rapid control in ruptured abdominal aneurysms. Arch Surg. 1981;116(8):1034-6.

66. Sovik E, Stokkeland P, Storm BS, Asheim P, Bolas O. The use of aortic occlusion balloon catheter without fluoroscopy for life-threatening post-partum haemorrhage. Acta Anaesthesiol Scand. 2012;56(3):388-93. https://doi.org/10.111 1/j.1399-6576.2011.02611.x.

67. Taheri SA, Mueller GA. Transaortic occlusion for aortic resection. Case reports. Vasc Surg. 1988;22(3):201-4.

68. Wang G, Zhou D, Shen WJ, Xin M, He J, Li Q, et al. Management of partial traumatic hemipelvectomy. Orthopedics. 2013;36(11):e1340-5. https://doi.org/10.3928/01477447-20131 $021-12$.

69. Xue-Song L, Chao Y, Kai-Yong Y, Si-Qing H, Heng Z. Surgical excision of extensive sacrococcygeal chordomas assisted by occlusion of the abdominal aorta. J Neurosurg Spine. 2010;12(5):490-6. https://doi.org/10.3171/2009.11.SPINE0977.

70. Yang L, Chong-Qi T, Hai-Bo S, Lan Z, Tian-Fu Y, Hong D, et al. Appling the abdominal aortic-balloon occluding combine with blood pressure sensor of dorsal artery of foot to control bleeding during the pelvic and sacrum tumors surgery. J Surg Oncol. 2008;97(7):626-8. https://doi.org/10.1002/jso.21026.

71. Zhang L, Gong Q, Xiao H, Tu C, Liu J. Control of blood loss during sacral surgery by aortic balloon occlusion. Anesth Analg. 2007;105(3):700-3. https://doi.org/10.1213/01.ane.0000278135 $85206.4 \mathrm{e}$.

72. Alsac JM, Desgranges P, Kobeiter H, Becquemin JP. Emergency endovascular repair for ruptured abdominal aortic aneurysms: feasibility and comparison of early results with conventional open repair. Eur J Vasc Endovasc Surg. 2005;30(6):632-9. https ://doi.org/10.1016/j.ejvs.2005.06.010.

73. Anain PM, Anain JM, Sr., Tiso M, Nader ND, Dosluoglu $\mathrm{HH}$. Early and mid-term results of ruptured abdominal aortic aneurysms in the endovascular era in a community hospital. J Vasc Surg. 2007;46(5):898-905. https://doi.org/10.1016/j. jvs.2007.06.037.

74. Carrafiello G, Piffaretti G, Lagana D, Fontana F, Mangini M, Ierardi AM, et al. Endovascular treatment of ruptured abdominal aortic aneurysms: aorto-uni-iliac or bifurcated endograft? Radiol Med. 2012;117(3):410-25. https://doi.org/10.1007/s1154 7-011-0717-2.

75. Coppi G, Silingardi R, Gennai S, Saitta G, Ciardullo AV. A single-center experience in open and endovascular treatment of hemodynamically unstable and stable patients with ruptured abdominal aortic aneurysms. J Vasc Surg. 2006;44(6):1140-7. https://doi.org/10.1016/j.jvs.2006.08.070. 
76. Dalainas I, Nano G, Bianchi P, Stegher S, Casana R, Malacrida $\mathrm{G}$, et al. Endovascular techniques for the treatment of ruptured abdominal aortic aneurysms: 7-year intention-to-treat results. World J Surg. 2006;30(10):1809-14. https://doi.org/10.1007/ s00268-005-0667-8 (discussion 15-6).

77. Djavani Gidlund K, Wanhainen A, Bjorck M. Intra-abdominal hypertension and abdominal compartment syndrome after endovascular repair of ruptured abdominal aortic aneurysm. Eur J Vasc Endovasc Surg. 2011;41(6):742-7. https://doi. org/10.1016/j.ejvs.2011.02.021.

78. Gerassimidis TS, Karkos CD, Karamanos DG, Papazoglou KO, Papadimitriou DN, Demiropoulos FP, et al. Endovascular management of ruptured abdominal aortic aneurysms: an 8 -year single-centre experience. Cardiovasc Intervent Radiol. 2009;32(2):241-9. https://doi.org/10.1007/s00270-008-9441-2.

79. Holst J, Resch T, Ivancev K, Bjorses K, Dias N, Lindblad B, et al. Early and intermediate outcome of emergency endovascular aneurysm repair of ruptured infrarenal aortic aneurysm: a single-centre experience of 90 consecutive patients. Eur J Vasc Endovasc Surg. 2009;37(4):413-9. https://doi.org/10.1016/j. ejvs.2008.12.015.

80. Ioannidis O, Trellopoulos G, Tamouridis G, Konstantinidis K, Megalopoulos A. A single-centre experience of the treatment of ruptured abdominal aortic aneurysms: clinical and anatomic characteristics of open versus endovascular repair. Int Angiol. 2012;31(4):386-92.

81. Karkos CD, Karamanos D, Papazoglou KO, Kantas AS, Theochari EG, Kamparoudis AG, et al. Usefulness of the Hardman index in predicting outcome after endovascular repair of ruptured abdominal aortic aneurysms. J Vasc Surg. 2008;48(4):788-94. https://doi.org/10.1016/j.jvs.2008.05.065.

82. Larzon T, Lindgren R, Norgren L. Endovascular treatment of ruptured abdominal aortic aneurysms: a shift of the paradigm? J Endovasc Ther. 2005;12(5):548-55. https://doi. org/10.1583/04-1469.1.

83. Low RB, Longmore W, Rubinstein R, Flores L, Wolvek S. Preliminary report on the use of the Percluder occluding aortic balloon in human beings. Ann Emerg Med. 1986;15(12):1466-9.

84. Luo Y, Duan H, Liu W, Min L, Shi R, Zhang W, et al. Clinical evaluation for lower abdominal aorta balloon occluding in the pelvic and sacral tumor resection. J Surg Oncol. 2013;108(3):148-51. https://doi.org/10.1002/jso.23376.

85. Mayer D, Aeschbacher S, Pfammatter T, Veith FJ, Norgren L, Magnuson A, et al. Complete replacement of open repair for ruptured abdominal aortic aneurysms by endovascular aneurysm repair: a two-center 14-year experience. Ann Surg. 2012;256(5):688-95. https://doi.org/10.1097/SLA.0b013e3182 71cebd (discussion 95-6).

86. Mehta M, Darling RC, 3rd, Roddy SP, Fecteau S, Ozsvath KJ, Kreienberg PB, et al. Factors associated with abdominal compartment syndrome complicating endovascular repair of ruptured abdominal aortic aneurysms. J Vasc Surg. 2005;42(6):1047-51. https://doi.org/10.1016/j. jvs.2005.08.033

87. Mehta M, Paty PS, Byrne J, Roddy SP, Taggert JB, Sternbach $\mathrm{Y}$, et al. The impact of hemodynamic status on outcomes of endovascular abdominal aortic aneurysm repair for rupture. J Vasc Surg. 2013;57(5):1255-60. https://doi.org/10.1016/j. jvs.2012.11.042.

88. Moore LJ, Brenner M, Kozar RA, Pasley J, Wade CE, Baraniuk MS, et al. Implementation of resuscitative endovascular balloon occlusion of the aorta as an alternative to resuscitative thoracotomy for noncompressible truncal hemorrhage. J Trauma Acute Care Surg. 2015;79(4):523-30. https://doi.org/10.1097/ TA.0000000000000809 (discussion 30-2).
89. Moore R, Nutley M, Cina CS, Motamedi M, Faris P, Abuznadah W. Improved survival after introduction of an emergency endovascular therapy protocol for ruptured abdominal aortic aneurysms. J Vasc Surg. 2007;45(3):443-50. https://doi. org/10.1016/j.jvs.2006.11.047.

90. Mukherjee D, Kfoury E, Schmidt K, Waked T, Hashemi H. Improved results in the management of ruptured abdominal aortic aneurysm may not be on the basis of endovascular aneurysm repair alone. Vascular. 2014;22(1):51-4.

91. Nedeau AE, Pomposelli FB, Hamdan AD, Wyers MC, Hsu R, Sachs T, et al. Endovascular vs open repair for ruptured abdominal aortic aneurysm. J Vasc Surg. 2012;56(1):15-20. https://doi. org/10.1016/j.jvs.2011.12.067.

92. Norii T, Crandall C, Terasaka Y. Survival of severe blunt trauma patients treated with resuscitative endovascular balloon occlusion of the aorta compared with propensity score-adjusted untreated patients. J Trauma Acute Care Surg. 2015;78(4):721-8. https:// doi.org/10.1097/TA.0000000000000578.

93. Ockert S, Schumacher H, Bockler D, Megges I, Allenberg JR. Early and midterm results after open and endovascular repair of ruptured abdominal aortic aneurysms in a comparative analysis. J Endovasc Ther. 2007;14(3):324-32. https://doi. org/10.1583/06-2065.1.

94. Peppelenbosch N, Geelkerken RH, Soong C, Cao P, Steinmetz OK, Teijink JA, et al. Endograft treatment of ruptured abdominal aortic aneurysms using the Talent aortouniiliac system: an international multicenter study. J Vasc Surg. 2006;43(6):1111-23. https://doi.org/10.1016/j.jvs.2006.01.035 (discussion 23).

95. Raux M, Marzelle J, Kobeiter H, Dhonneur G, Allaire E, Cochennec F, et al. Endovascular balloon occlusion is associated with reduced intraoperative mortality of unstable patients with ruptured abdominal aortic aneurysm but fails to improve other outcomes. J Vasc Surg. 2015;61(2):304-8. https://doi.org/10.1016/j. jvs.2014.07.098

96. Resch T, Malina M, Lindblad B, Dias NV, Sonesson B, Ivancev K. Endovascular repair of ruptured abdominal aortic aneurysms: logistics and short-term results. J Endovasc Ther. 2003;10(3):440-6. https://doi.org/10.1177/152660280301000 307.

97. Saito N, Matsumoto H, Yagi T, Hara Y, Hayashida K, Motomura $\mathrm{T}$, et al. Evaluation of the safety and feasibility of resuscitative endovascular balloon occlusion of the aorta. J Trauma Acute Care Surg. 2015;78(5):897-903. https://doi.org/10.1097/ TA.0000000000000614 (discussion 4).

98. Sarac TP, Bannazadeh M, Rowan AF, Bena J, Srivastava S, Eagleton M, et al. Comparative predictors of mortality for endovascular and open repair of ruptured infrarenal abdominal aortic aneurysms. Ann Vasc Surg. 2011;25(4):461-8. https://doi. org/10.1016/j.avsg.2010.12.030.

99. Starnes BW, Quiroga E, Hutter C, Tran NT, Hatsukami T, Meissner M, et al. Management of ruptured abdominal aortic aneurysm in the endovascular era. J Vasc Surg. 2010;51(1):9-17. https:// doi.org/10.1016/j.jvs.2009.08.038 (discussion 8).

100. Takagi H, Hirose H, Mori Y, Iwata H, Umeda Y, Matsuno Y. Antegradely insertable aortic balloon occlusion catheter for aortic arch repair. Heart Vessels. 2003;18(2):75-8. https://doi. org/10.1007/s10380-002-0688-3.

101. Tang X, Guo W, Yang R, Tang S, Dong S. Use of aortic balloon occlusion to decrease blood loss during sacral tumor resection. J Bone Joint Surg Am. 2010;92(8):1747-53. https://doi. org/10.2106/JBJS.I.01333.

102. Veith FJ, Ohki T. Endovascular approaches to ruptured infrarenal aorto-iliac aneurysms. J Cardiovasc Surg (Torino). 2002;43(3):369-78.

103. Veith FJ, Ohki T, Lipsitz EC, Suggs WD, Cynamon J. Endovascular grafts and other catheter-directed techniques in the 
management of ruptured abdominal aortic aneurysms. Semin Vasc Surg. 2003;16(4):326-31

104. Matsumura Y, Matsumoto J, Kondo H, Idoguchi K, Funabiki T. investigators D-I. Partial occlusion, conversion from thoracotomy, undelayed but shorter occlusion: resuscitative endovascular balloon occlusion of the aorta strategy in Japan. Eur J Emerg Med. 2017. https://doi.org/10.1097/MEJ.0000000000000466.

105. Sadeghi M, Nilsson KF, Larzon T, Pirouzram A, Toivola A, Skoog P, et al. The use of Aortic Balloon Occlusion in traumatic shock- first report from the ABO Trauma Registry. Eur J Trauma. 2017. Accepted.

106. Badger SA, Harkin DW, Blair PH, Ellis PK, Kee F, Forster R. Endovascular repair or open repair for ruptured abdominal aortic aneurysm: a Cochrane systematic review. BMJ Open. 2016;6(2):e008391. https://doi.org/10.1136/bmjopen-2015008391.

107. Harkin DW, Dillon M, Blair PH, Ellis PK, Kee F. Endovascular ruptured abdominal aortic aneurysm repair (EVRAR): a systematic review. Eur J Vasc Endovasc Surg. 2007;34(6):673-81. https ://doi.org/10.1016/j.ejvs.2007.06.004.

108. Hinchliffe RJ, Braithwaite BD, Hopkinson BR. The endovascular management of ruptured abdominal aortic aneurysms. Eur J Vasc Endovasc Surg. 2003;25(3):191-201. https://doi.org/10.1053/ ejvs.2002.1846.

109. Karkos CD, Harkin DW, Giannakou A, Gerassimidis TS. Mortality after endovascular repair of ruptured abdominal aortic aneurysms: a systematic review and meta-analysis. Arch Surg. 2009;144(8):770-8. https://doi.org/10.1001/archsurg.2009.132.

110. Karkos CD, Sutton AJ, Bown MJ, Sayers RD. A meta-analysis and metaregression analysis of factors influencing mortality after endovascular repair of ruptured abdominal aortic aneurysms. Eur J Vasc Endovasc Surg. 2011;42(6):775-86. https:// doi.org/10.1016/j.ejvs.2011.07.028.

111. DuBose JJ, Scalea TM, Brenner M, Skiada D, Inaba K, Cannon $\mathrm{J}$, et al. The AAST prospective aortic occlusion for resuscitation in trauma and acute care surgery (AORTA) registry: data on contemporary utilization and outcomes of aortic occlusion and resuscitative balloon occlusion of the aorta (REBOA). J Trauma Acute Care Surg. 2016;81(3):409-19. https://doi.org/10.1097/ TA.0000000000001079.

112. Champion HR, Bellamy RF, Roberts CP, Leppaniemi A. A profile of combat injury. J Trauma. 2003;54(5 Suppl):S13-9. https ://doi.org/10.1097/01.TA.0000057151.02906.27.

113. Holcomb JB, McMullin NR, Pearse L, Caruso J, Wade CE, Oetjen-Gerdes L, et al. Causes of death in US Special Operations Forces in the global war on terrorism: 2001-2004. Ann Surg. 2007;245(6):986-91. https://doi.org/10.1097/01.sla.00002 59433.03754.98.

114. Belmont PJ Jr, Goodman GP, Zacchilli M, Posner M, Evans C, Owens BD. Incidence and epidemiology of combat injuries sustained during "the surge" portion of operation Iraqi Freedom by a US Army brigade combat team. J Trauma. 2010;68(1):204-10. https://doi.org/10.1097/TA.0b013e3181bdcf95.

115. Kelly JF, Ritenour AE, McLaughlin DF, Bagg KA, Apodaca AN, Mallak CT, et al. Injury severity and causes of death from Operation Iraqi Freedom and Operation Enduring Freedom: 2003-2004 versus 2006. The Journal of trauma. 2008;64(2 Suppl):S21-6. https://doi.org/10.1097/TA.0b013e318160b9fb (discussion S6-7).

116. Desgranges P. Latest news from the ECAR randomised controlled trial. New York, USA: Veith symposium. 2013.

117. Desgranges P. Latest news from the ECAR randomised controlled trial. Paris: Controversies and Updates in Vascular Surgery; 2014.

118. Desgranges P, Kobeiter H, Katsahian S, Bouffi M, Gouny P, Favre JP et al. Editor's choice-ECAR (Endovasculaire ou
Chirurgie dans les Anevrysmes aorto-iliaques Rompus): a French Randomized Controlled Trial of Endovascular Versus Open Surgical Repair of Ruptured Aorto-iliac Aneurysms. Eur J Vasc Endovasc Surg. 2015;50(3):303-10. https://doi.org/10.1016/j. ejvs.2015.03.028.

119. Investigators IT, Powell JT, Sweeting MJ, Thompson MM, Ashleigh R, Bell R, et al. Endovascular or open repair strategy for ruptured abdominal aortic aneurysm: 30 day outcomes from IMPROVE randomised trial. BMJ. 2014;348:f7661. https://doi. org/10.1136/bmj.f7661.

120. Reimerink JJ, Hoornweg LL, Vahl AC, Wisselink W, van den Broek TA, Legemate DA, et al. Endovascular repair versus open repair of ruptured abdominal aortic aneurysms: a multicenter randomized controlled trial. Ann Surg. 2013;258(2):248-56. https://doi.org/10.1097/SLA.0b013e3182 $8 \mathrm{~d} 4 \mathrm{~b} 76$.

121. Badger S, Bedenis R, Blair PH, Ellis P, Kee F, Harkin DW. Endovascular treatment for ruptured abdominal aortic aneurysm. Cochrane Database Syst Rev. 2014(7):CD005261. https ://doi.org/10.1002/14651858.CD005261.pub3.

122. Gamberini E, Coccolini F, Tamagnini B, Martino C, Albarello $\mathrm{V}$, Benni M, et al. Resuscitative endovascular balloon occlusion of the aorta in trauma: a systematic review of the literature. World J Emerg Surg. 2017;12:42. https://doi.org/10.1186/ s13017-017-0153-2.

123. Linsenmaier U, Kanz KG, Rieger J, Krotz M, Mutschler W, Pfeifer KJ, et al. CT-guided aortic balloon occlusion in traumatic abdominal and pelvic bleeding. Rofo. 2003;175(9):125963. https://doi.org/10.1055/s-2003-41931.

124. Costantini TW, Coimbra R, Holcomb JB, Podbielski JM, Catalano R, Blackburn A, et al. Current management of hemorrhage from severe pelvic fractures: Results of an American Association for the Surgery of Trauma multi-institutional trial. J Trauma Acute Care Surg. 2016;80(5):717-23. https://doi. org/10.1097/TA.0000000000001034 (discussion 23-5).

125. Long JA, Descotes JL, Terrier N, Faucheron JL, Pecher M, Francony G, et al. Endovascular aortic balloon catheter occlusion for severe renal trauma. Prog Urol. 2004;14(3):394-7 (discussion 7).

126. Okada Y, Narumiya H, Ishi W, Ryoji I. Lower limb ischemia caused by resuscitative balloon occlusion of aorta. Surg Case Rep. 2016;2(1):130. https://doi.org/10.1186/s4079 2-016-0260-4.

127. Segol P, Salame E, Auvray S, Marchand P, Tartiere J. Major liver injury. Role of preoperative transcutaneous endoluminal aortic clamping. Presse Med. 1995;24(1):29-30.

128. Uchino H, Tamura N, Echigoya R, Ikegami T, Fukuoka T. "REBOA"-is it really safe? A case with massive intracranial hemorrhage possibly due to endovascular balloon occlusion of the aorta (REBOA). Am J Case Rep. 2016;17:810-3.

129. Sadek S, Lockey DJ, Lendrum RA, Perkins Z, Price J, Davies GE. Resuscitative endovascular balloon occlusion of the aorta (REBOA) in the pre-hospital setting: an additional resuscitation option for uncontrolled catastrophic haemorrhage. Resuscitation. 2016;107:135-8. https://doi.org/10.1016/j.resuscitat ion.2016.06.029.

130. Tsurukiri J, Akamine I, Sato T, Sakurai M, Okumura E, Moriya $\mathrm{M}$, et al. Resuscitative endovascular balloon occlusion of the aorta for uncontrolled haemorrahgic shock as an adjunct to haemostatic procedures in the acute care setting. Scand J Trauma Resusc Emerg Med. 2016;24:13. https://doi.org/10.1186/s1304 9-016-0205-8.

131. Inoue J, Shiraishi A, Yoshiyuki A, Haruta K, Matsui H, Otomo Y. Resuscitative endovascular balloon occlusion of the aorta might be dangerous in patients with severe torso trauma: a propensity score analysis. J Trauma Acute Care Surg. 2016;80(4):559-66. 
https://doi.org/10.1097/TA.0000000000000968 (discussion 66-7).

132. Kataoka Y, Minehara H, Shimada K, Nishimaki H, Soma K, Maekawa K. Sepsis caused by peripelvic soft tissue infections in critically injured patients with multiple injuries and unstable pelvic fracture. J Trauma. 2009;66(6):1548-54. https://doi. org/10.1097/TA.0b013e3181a51b0e. discussion 54-5.

133. Teeter WA, Matsumoto J, Idoguchi K, Kon Y, Orita T, Funabiki $\mathrm{T}$, et al. Smaller introducer sheaths for REBOA may be associated with fewer complications. J Trauma Acute Care Surg. 2016;81(6):1039-45. https://doi.org/10.1097/TA.0000000000 001143.

134. Morrison JJ, Ross JD, Markov NP, Scott DJ, Spencer JR, Rasmussen TE. The inflammatory sequelae of aortic balloon occlusion in hemorrhagic shock. J Surg Res. 2014;191(2):423-31. https://doi.org/10.1016/j.jss.2014.04.012.

135. Reva VA, Matsumura Y, Horer T, Sveklov DA, Denisov AV, Telickiy SY, et al. Resuscitative endovascular balloon occlusion of the aorta: what is the optimum occlusion time in an ovine model of hemorrhagic shock? Eur J Trauma Emerg Surg. 2016. https:// doi.org/10.1007/s00068-016-0732-z.
136. Morrison JJ, Reva V, Lönn L, Matsumoto J, Matsumara Y, Holcomb J, et al. Resuscitative Endovascular Balloon Occlusion of the Aorta (REBOA). In: Hörer TM, editor. Top stent: the art of endovascular hybrid Trauma and bleeding management. Örebro: Örebro University Hospita; 2015. pp. 77-100.

137. American Association for the Surgery of Trauma. Multi-Institutional Studies. 2017. http://www.aast.org/Research/MultiInsti tutionalStudies.aspx. Accessed 16 July 2017.

138. ABO Trauma Registry. ABO trauma registry. 2017. https://www. abotraumaregistry.com. Accessed 16 July 2017.

139. Society of DIRECT. DIRECT-IABO Registry. 2017. http://direc t.kenkyuukai.jp/about/. Accessed 16 July 2017.

140. Jansen J, Campbell M, Cochran C, Clancy A. The UK-REBOA Trial. 2017. https://w3.abdn.ac.uk/hsru/REBOA/Public/Public/ index.cshtml. Accessed 16 July 2017.

141. DuBose JJ. How I do it: partial resuscitative endovascular balloon occlusion of the aorta (P-REBOA). J Trauma Acute Care Surg. 2017;83(1):197-9. https://doi.org/10.1097/TA.0000000000 001462 .

\section{Affiliations}

\section{B. L. S. Borger van der Burg ${ }^{1}$ - Thijs T. C. F. van Dongen ${ }^{1,2}{ }^{(1)}$. J. J. Morrison ${ }^{3} \cdot$ P. P. A. Hedeman Joosten ${ }^{1}$. J. J. DuBose ${ }^{4}$. T. M. Hörer ${ }^{5}$ R. Hoencamp ${ }^{1,2,6}$}

B. L. S. Borger van der Burg blsborgervanderburg@alrijne.nl

J. J. Morrison

jonathan.morrison@umm.edu

P. P. A. Hedeman Joosten ppahedemanjoosten@alrijne.nl

J. J. DuBose

jjd3c@yahoo.com

T. M. Hörer

tal.horer@regionorebrolan.se

R. Hoencamp

r.hoencamp@lumc.nl
1 Department of Surgery, Alrijne Hospital, Simon Smitweg 1, 2353 GA Leiderdorp, The Netherlands

2 Defense Healthcare Organization, Ministry of Defense, Utrecht, The Netherlands

3 R. Adam Cowley Shock Trauma Center, University of Maryland Medical System, Baltimore, USA

4 Division of Vascular Surgery, David Grant Medical Center, Travis AFB, California, USA

5 Department of Cardiothoracic and Vascular Surgery, Örebro University Hospital, Örebro University, Örebro, Sweden

6 Division of Surgery, Leiden University Medical Centre, Leiden, The Netherlands 